CLINICAL STUDY

\title{
Clinical and biological characterization of macroprolactinemia with and without prolactin-IgG complexes
}

Jean De Schepper, Johan Schiettecatte ${ }^{2}$, Brigitte Velkeniers, Zeev Blumenfeld ${ }^{3}$, Michal Shteinberg ${ }^{3}$, Paul Devroey ${ }^{1}$, Ellen Anckaert ${ }^{2}$, Johan Smitz ${ }^{2}$, Peggy Verdood ${ }^{4}$, Robert Hooghe ${ }^{4}$ and Elisabeth Hooghe-Peters ${ }^{4}$

Department of Endocrinology, ${ }^{1}$ Fertility Clinic and ${ }^{2}$ Endocrine Laboratory of the Academic Hospital VUB, Brussels, Belgium,

${ }^{3}$ Reproductive Endocrinology Section, Department of Obstetrics/Gynecology, Rambam Medical Center, Faculty of Medicine, Technion,

Israel Institute of Technology, Haifa, Israel and ${ }^{4}$ Laboratory of Pharmacology of the Medical Faculty of the Free University of Brussels, Brussels, Belgium

(Correspondence should be addressed to J De Schepper, Academic Hospital VUB, Laarbeeklaan, 101, 1090 Brussels; Belgium;

Email: jean.deschepper@az.vub.ac.be)

\begin{abstract}
Objective: Macroprolactinemia, which can be detected by a polyethylene glycol (PEG) precipitation test, is a clinically and biologically heterogeneous condition. In this study, we analyzed whether the clinical presentation, the hormonal findings and the in vitro lactogenic activity differed between macroprolactinemic patients with and without circulating prolactin (PRL) - IgG complexes.

Design: Clinical data were reviewed and additional hormonal studies were performed in 50 hyperprolactinemic patients with macroprolactinemia.

Methods: Macroprolactinemia was identified by a PRL recovery after PEG precipitation of $<50 \%$, as measured by an automated commercial immunoassay system and circulating PRL-IgG complexes by an abnormal PRL binding to anti-IgG agarose.

Results: PRL-IgG complexes were found in 46 patients. The origin of hyperprolactinemia in these 46 patients was idiopathic in 33 patients, while a pituitary lesion or stalk magnetic resonance imaging or computed tomography scan was detected in 13 patients found compression. Galactorrhea was found in 11 of these 46 patients, while this condition was present in three of the four patients without circulating PRL-IgG complexes. The median free PRL concentration was significantly lower in patients with PRL-IgG complexes than in the group without complexes $(243$ vs $969 \mathrm{mIU} / \mathrm{l} ; P<0.005)$, whereas median total PRL immunoreactivity and median PRL bioactivity in the $\mathrm{Nb} 2$ assay were not significantly different. In patients with circulating PRL-IgG complexes, $\mathrm{Nb} 2$ bioassay results correlated significantly with total PRL immunoreactivity $(r=0.64 ; P<0.0001)$, but not with free PRL results $(r=0.24 ; P<0.17)$.

Conclusions: These results indicate that PRL-IgG complexes (i) account for most cases of macroprolactinemia - as identified by PEG precipitation - in hyperprolactinemic patients presenting with a variety of diagnoses, (ii) are not associated with a specific clinical presentation, (iii) can be found in patients with diverse pituitary pathologies, and (iv) possess an in vitro lactogenic activity in the $\mathrm{Nb} 2$ bioassay in relation to their immunoreactivity.
\end{abstract}

European Journal of Endocrinology 149 201-207

\section{Introduction}

Prolactin (PRL) circulates in human serum in forms of different molecular sizes. In normal sera, the major form is the monomeric form with a molecular mass of $23 \mathrm{kDa}$, but in some sera a form with a molecular mass $>150 \mathrm{kDa}$ (named big, big PRL) is predominant $(1-4)$. This phenomenon is called macroprolactinemia and is classically identified by gel filtration chromatography, a rather time-consuming and rather difficult technique. Recently the reliability of the polyethylene glycol (PEG) precipitation test for the routine detection of macroprolactinemia in hyperprolactinemic samples by different immunoassays has been established (4-8). The prevalence of macroprolactinemia in subjects with hyperprolactinemia using this method was found to be between 9 and $26 \%$ in prospective laboratory studies $(4-10)$.

The term macroprolactin designates as well PRLimmunoglobulin G (IgG) complexes as covalently or non-covalently bound aggregates of monomeric PRL molecules (3, 11-14). Recent evidence from larger studies, however, indicates that macroprolactinemia is mostly due to PRL-IgG complexes $(10,15,16)$. Despite renewed interest in this condition, it remains so far unclear whether the nature of the complexes 
(PRL-IgG complexes versus PRL polymers) affects PRL bioactivity and clinical presentation.

The clinical correlates of the presence of a high molecular mass form of immunoassayable PRL in the blood circulation show great diversity between patients. Many patients lack the clinical manifestations typical of the hyperprolactinemia syndrome, while others with similar serum levels of PRL have complaints such as oligomenorrhea, minimal lactation, gynecomastia or impotence $(5,9,15-17)$. Therefore, we designed the present study (i) to determine the prevalence of PRL-IgG complexes in hyperprolactinemic patients with macroprolactinemia (detected by PEG precipitation), (ii) to analyze whether the clinical and hormonal findings differ between macroprolactinemic patients with and without circulating PRL-IgG complexes, and (iii) to explore whether these complexes possess lactogenic activity and whether the level of PRL bioactivity in vitro is related to the clinical presentation and/or the PRL immunoreactivity.

\section{Subjects and methods}

Clinical data were reviewed and additional biochemical studies were performed in 50 consecutive hyperprolactinemic patients with macroprolactinemia, identified by a PRL recovery after PEG precipitation of $<50 \%$, as measured by an automated commercial electrochemiluminescent immunoassay system (Elecsys; Roche Diagnostics, Mannheim, Germany) (10). These patients were detected by the systematic performance of a PEG precipitation when the serum PRL value exceeded $1000 \mathrm{mIU} / \mathrm{l}$. The presence of macroprolactinemia was confirmed by gel filtration chromatography in serum samples of patients with a recovery between 40 and $50 \%$, since these cut-offs have been considered in some studies as a gray zone, where the presence of macroprolactinemia has to be confirmed or refuted by chromatography $(5,7)$. Forty-two patients were followed at the Clinics of Endocrinology and Fertility of the Academic Hospital of the Free University of Brussels and eight at the Department of Obstetrics and Gynecology, Rambam Medical Center, Haifa, Israel. One patient had liver cirrhosis and three of the subjects were taking PRL-raising medication. Idiopathic growth hormone deficiency of childhood-onset had been diagnosed in one patient and one patient had been operated for a macroprolactinoma. Parasellar meningioma (one case), non-secreting gonadotropinoma (one case) and cerebral sarcoidosis (one case) were diagnosed after the initial screening procedure.

Clinical data collected in these 50 macroprolactinemic subjects were age and sex of the patients, reason for PRL determination, presence or absence of symptoms related to the hyperprolactinemia syndrome (infertility, oligoamenorrhea and galactorrhea in women; galactorrhea and impotence or loss of libido in men), results of pituitary gland imaging, diagnosis of hyperprolactinemia and presence or absence of another pathology explaining the presenting symptomatology (Table 1).

If not yet available at the time of the PRL measurement, serum concentrations of luteinizing hormone, follicle-stimulating hormone, estradiol (in women), testosterone (in men), free thyroxine, thyrotropin and anti-thyroid peroxidase antibodies were measured in all sera by standard automated immunoassays. All PRL measurements in this study were done with the automated Elecsys PRL assay, which uses the electrochemiluminescent reaction of $\mathrm{Ru}$ (II) Tris (bipyridil) with tripropylamine combined with magnetic microparticle separation. This PRL assay has a dual monoclonal sandwich format with an incubation time of $18 \mathrm{~min}$. The sample volume is $10 \mu \mathrm{l}$ and the measuring range is $10-10000 \mathrm{mIU} / \mathrm{l}$ (Third IRP WHO Reference Standard 84/500). The limit of detection is $3.2 \mathrm{mIU} / \mathrm{l}$. The within-run and between-run coefficient of variation (CV) are $<3.1 \%$ and $<4.0 \%$ (range $130-6800 \mathrm{mIU} / \mathrm{l}$ ) respectively. All PEG precipitation tests and gel filtration chromatographs were done at the Endocrine Laboratory (Academic Hospital, Free University of Brussels, Belgium) as described previously (10). A free PRL or nonPEG precipitable PRL immunoreactivity, defined by the product of serum PRL with the percentage recovery after PEG precipitation, was calculated. In an attempt to characterize the nature of the high molecular mass forms of PRL, we performed an immunoprecipitation test with anti-human IgG agarose, as described previously (10). This method has, in our laboratory, a between-run $\mathrm{CV}$ of $3.5 \%$. A cut-off of $15 \%$, presenting the highest binding of non-macroprolactin-containing

Table 1 Clinical and biological characteristics of the different diagnostic groups with macroprolactinemia.

\begin{tabular}{lccccccc}
\hline $\begin{array}{l}\text { Diagnosis of } \\
\text { hyperprolactinemia }\end{array}$ & $\begin{array}{c}\text { Total } \\
\text { number }\end{array}$ & $\begin{array}{c}\text { Sex ratio } \\
\text { (female/male) }\end{array}$ & $\begin{array}{c}\text { Median age } \\
\text { (years) }\end{array}$ & $\begin{array}{c}\text { Number with } \\
\text { alternative diagnosis } \\
\text { for complaint }\end{array}$ & $\begin{array}{c}\text { Number with } \\
\text { PRL-IgG } \\
\text { complexes }\end{array}$ & $\begin{array}{c}\text { Median } \\
\text { serum PRL } \\
\text { (mlU/l) }\end{array}$ & $\begin{array}{c}\text { Median serum } \\
\text { free PRL } \\
\text { (mlU/l) }\end{array}$ \\
\hline Idiopathic hyperprolactinemia & 35 & $31 / 4$ & 35 & 15 & 33 & 2100 & 234 \\
Microprolactinoma & 8 & $8 / 0$ & 32 & 1 & 8 & 2060 & 232 \\
Residual macroprolactinoma & 1 & $1 / 0$ & 83 & 0 & 1 & 2069 & 167 \\
Stalk compression & 3 & $2 / 1$ & 33 & 1 & 2 & 2824 & 724 \\
Drug-induced & 3 & $2 / 1$ & 29 & 0 & 2 & 3954 & 825 \\
\hline
\end{tabular}


sera, was used to identify the presence of PRL-IgG complexes. In 37 of the patients, serum was available for a bioassay of lactogenic activity, using $\mathrm{Nb} 2$ cells, as described previously (18). The sensitivity of the assay is, in our hands, $150 \mathrm{mIU} / \mathrm{l}$. Statistical analysis included the Mann-Whitney or Kruskal-Wallis tests for comparisons of continuous parameters and a chi-square test for categorical parameters.

\section{Results}

Forty-four subjects with macroprolactinemia were females with a median age of 34.5 years at presentation (range 14-83 years) and six were males with a median age of 41.5 years (range 13-63 years). In females the initial complaint and reason for performing a PRL measurement was primary infertility in 17 , secondary infertility in 11, galactorrhea in five, loss of libido in two and secondary amenorrhea in three. In two females, a PRL measurement was done in the endocrine follow-up of a known PRL-secreting pituitary adenoma and in four subjects as part of a general hormonal screening for a suspected or known pituitary pathology. In male patients, the clinical incentive for PRL determination was galactorrhea in two, gynecomastia in three and loss of libido in one. Galactorrhea was found at clinical examination in 14 patients (two males and 12 females) and oligomenorrhea was documented in six premenopausal women. Loss of libido was an ancillary complaint in three (two females and one male) and mastodynia was noted in three women. Of the 35 patients who had a pituitary imaging (ten had computed tomography (CT) scans and 25 magnetic resonance imaging (MRI)) performed in the first months after the detection of the hyperprolactinemia, a parasellar meningioma was found in one patient, a residual intrasellar mass (post-macroprolactinoma resection) in another and a small $(<10 \mathrm{~mm})$ intrapituitary lesion in 11. The diagnosis, based on endocrinological and radiographical examinations, in these 11 cases with a radiological pituitary anomaly were sarcoidosis (histologically confirmed) in one, gonadotropinoma in one, Rathke cyst in one, and a microprolactinoma in eight cases. In all men and premenopausal women serum concentrations of gonadotropins and sex steroids were normal. Thyroid function tests were normal in all patients, but three patients had positive anti-thyroid peroxidase antibodies.

As shown in Table 1, diagnosis of hyperprolactinemia was in 35 patients idiopathic hyperprolactinemia, defined by a normal hormonal work-up and a normal pituitary imaging, which was performed in cases of hyperprolactinemia above $5000 \mathrm{mIU} / \mathrm{l}$. Other diagnoses were microprolactinoma in eight patients, a drug-induced hyperprolactinemia in three, a stalk compression in three, and a residual macroprolactinoma in one; an alternative origin for the complaint, such as polycystic ovary syndrome, tubal infertility or male infertility, was present in 15 of the 35 patients with idiopathic hyperprolactinemia, in one of the eight patients with microprolactinoma and in one of the three patients with a diagnosis of stalk compression. Moderate to marked elevations of serum PRL, ranging between 1054 and 6466 mIU/l (median concentration of $2203 \mathrm{mIU} / \mathrm{l}$ ) were found at initial screening. Six patients (three with idiopathic hyperprolactinemia) had a serum PRL concentration above $5000 \mathrm{mIU} / \mathrm{l}$, but in only one of these patients was free PRL, as defined by the product of PRL with the percentage recovery after PEG precipitation, above $1000 \mathrm{IU} / \mathrm{l}$. Free PRL was above $1000 \mathrm{mIU} / \mathrm{l}$ in only 5 of 50 studied subjects. Median PRL and free PRL concentration were not significantly different between patients with and without galactorrhea, in women with and without menstrual disturbances or in men with and without gynecomastia. In the immunoprecipitation test with anti-IgG agarose, performed on all serum samples, PRL binding ranged between 2.8 and 91\% (median value $66 \%$ ). In the seven serum samples selected for gel filtration chromatography by their recovery between 40 and $50 \%$, the proportion of macroprolactin, as determined by analysis of the area under the PRL curve, was above $30 \%$ in all cases.

Circulating PRL-IgG complexes, as defined by PRL binding to anti-IgG agarose above $15 \%$, were found in 46 patients. The origin of hyperprolactinemia in these patients was considered idiopathic in 33 patients, drug-induced in two and caused by a microprolactinoma in eight and by stalk compression in two. Circulating PRL-IgG complexes were found in the three patients with circulating anti-thyroid peroxidase antibodies and in three of the five patients with free PRL concentration above $1000 \mathrm{mIU} / \mathrm{l}$. In the four patients without PRL-IgG complexes, the hyperprolactinemia was drug-induced in one patient, idiopathic in two and caused by a stalk effect in one. While galactorrhea was found in three of the four patients without PRLIgG complexes, this symptom was only present in 11 of the 46 with circulating PRL-IgG complexes $(P<0.005)$. Primary infertility had been the reason for PRL measurement in 17 and secondary infertility in 11 of the 46 patients with positive PRL-IgG complexes. In the patients with PRL-IgG complexes, the percentage of PRL binding to anti-IgG agarose was independent of age and the initial reason for PRL determination. PRL binding to anti-IgG agarose correlated significantly $(r=-0.74 ; P<0.0001)$ with the percentage of the PRL recovery after PEG precipitation (Fig. 1). The four patients without PRL-IgG complexes had similar median serum PRL values (2417 vs $2722 \mathrm{mIU} / \mathrm{l} ; \quad P=0.68)$, but significantly higher median free PRL concentrations (969 vs $243 \mathrm{mIU} / \mathrm{l}$; $P<0.005)$ than those with $\mathrm{PRL}-\mathrm{IgG}$ complexes in the circulation (Table 2). The proportion of macroprolactin assessed by gel chromatography in these four 


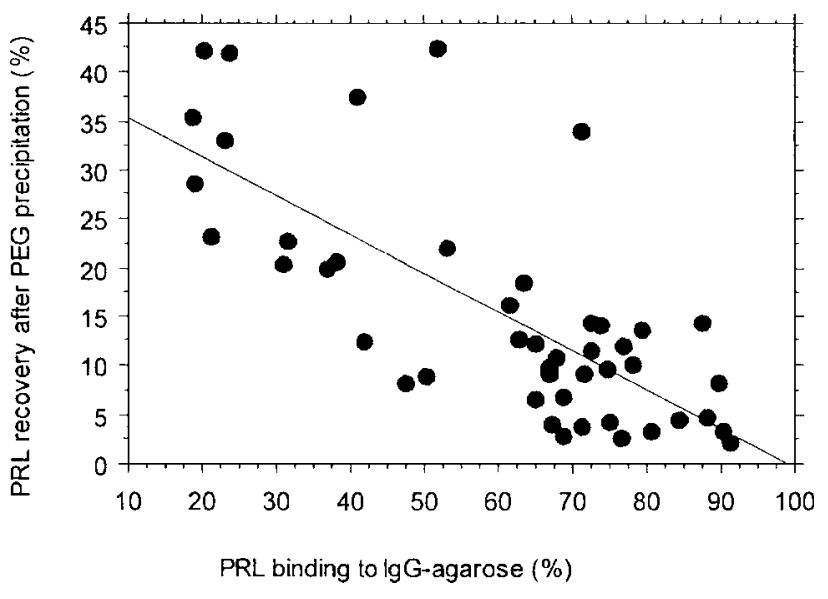

Figure 1 Scattergram of $P R L$ binding to $\lg G$ agarose in relation to $P R L$ recovery after $P E G$ precipitation in patients with $P R L-I g G$ complexes.

patients without PRL-IgG complexes was between 30 and $37 \%$.

Median PRL bioactivity in the $\mathrm{Nb} 2$ assay was not significantly different between patients with and without PRL-IgG complexes. Neither lower PRL bioactivity nor lower PRL immunoreactivity was present in the sera of those patients with an alternative diagnosis for their complaint. Median PRL bioactivity values did not differ between patients with or without typical hyperprolactinemia complaints such as galactorrhea, menstrual disturbances and infertility. In patients without circulating PRL-IgG complexes, PRL bioactivity values increased with increasing serum free PRL concentrations, but not with the total immunoassayable PRL content of the sera. In the patients with circulating PRL-IgG complexes, a significant correlation was found between bioactivity values and serum PRL concentrations $(r=0.64 ; P<0.0001)$, but not with the calculated free PRL values $(r=0.24 ; P=0.17)$ (Fig. 2).

\section{Discussion}

In the evaluation of patients with hyperprolactinemia, the performance of a PEG precipitation test has been advocated for serum samples in the hyperprolactinemic range in highly reacting commercial immunoassays, such as the Delphia and Elecsys systems $(6,8,19)$. Using this approach, we detected 50 patients with macroprolactinemia, which is one of the largest populations studied both clinically and biologically up to now. In accord with previous, albeit smaller studies, the majority of them were young to middle-aged adult females $(7,15,16)$. Menstrual disorders, infertility and galactorrhea are the classic symptoms observed in previous published series of women with macroprolactinemia $(7,9,15,16,20)$. Although most of the macroprolactinemic women in our survey consulted for infertility, this feature may have only initiated the PRL evaluation. Furthermore, its relationship to the hyperprolactinemia can be coincidental, given the presence of oligomenorrhea and amenorrhea in only a minority, an alternative diagnosis for their complaint in more than half of these hyperprolactinemic women, and the finding of normal gonadotropins and sexual steroids in all of them. Galactorrhea was another frequent complaint of both women and men with macroprolactinemia in our study. Neither serum total PRL immunoactivity nor free PRL values were found to be higher in these symptomatic patients. We cannot exclude, however, that because of a low stability of the high molecular mass PRL complexes, monomeric PRL levels are fluctuating and reach high levels, leading to clinical symptoms in some conditions $(13,21)$. A consistent tendency for monomeric PRL to increase faster than the high molecular mass PRL has been observed in macroprolactinemic women during pregnancy (21).

Since clinical symptoms were found to be correlated neither with total immunoreactive PRL nor with free PRL, we investigated other biological factors to explain the diversity in clinical symptomatology. Suspecting that part of the differential clinical presentation of macroprolactinemia between subjects might be related to the presence or absence of circulating PRL-IgG complexes, all macroprolactinemic samples were assessed by immunoprecipitation with anti-IgG agarose. Ninety-two percent of macroprolactinemic samples in our study were found to contain PRL-IgG complexes. Previous studies, investigating a rather small number of serum samples of macroprolactinemic women, have

Table 2 Comparison between PRL-IgG-positive and negative macroprolactinemic patients.

\begin{tabular}{lccc}
\hline & PRL-IgG-positive $(n=46)$ & PRL-lgG-negative $(n=4)$ & Significance \\
\hline Median age (years) & 34.5 & 45.5 & 0.186 \\
Sex (female/male) & $44 / 2$ & $2 / 2$ & 0.014 \\
Galactorrhea (yes/no) & $11 / 35$ & $3 / 1$ & 0.029 \\
Idiopathic (yes/no) & $33 / 13$ & $2 / 2$ & 0.362 \\
Median serum PRL (mIU/l) & 2203 & 2412 & 0.971 \\
Median serum free PRL(mIU/l) & 243 & 969 & 0.003 \\
\hline
\end{tabular}




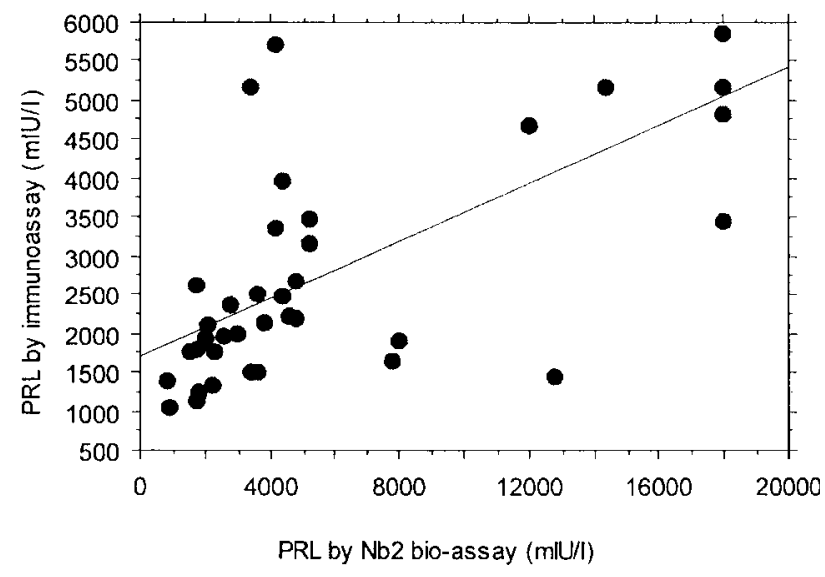

Figure 2 Scattergram of $P R L ~ N b 2$ bioactivity in relation to $P R L$ immunoreactivity.

shown that the majority of the high molecular mass PRL forms isolated by gel chromatography were retained on protein A or anti-IgG columns $(15,22)$. Since we identified only four patients without PRL-IgG complexes, it is difficult to make valid comparisons between patients with and without IgG in their PRL complexes. Galactorrhea, however, seemed to be more frequent in the group without PRL-IgG complexes. Although the free PRL concentration was in general higher in these four patients without PRLIgG complexes, it remained below the levels found mostly in patients with micro- or macroprolactinoma and was of no diagnostic aid in the discrimination between PRL-IgG complex-negative and -positive patients because of the great overlap of the values.

From a clinical point a view, symptomatic patients with mild to moderate hyperprolactinemia and/or idiopathic hyperprolactinemia, as was the case for many of our patients, present the greatest diagnostic difficulty. Serial biological and neuroradiological assessments have been recommended to identify the 'transition towards prolactinoma' (23). On the contrary, it has been proposed that the detection of PRL-IgG complexes in hyperprolactinemic sera eliminates the need for further investigations related to the hyperprolactinemia (7, 24). PRL- IgG complexes as well as PRL polymers have been found, however, in patients with prolactinoma, including macroprolactinoma $(16,22)$. In the present study, despite the fact that MRIs or CT scans were not included in the standard investigation protocol of moderately elevated serum PRL concentrations, pituitary abnormalities were documented in one-third of patients with circulating PRL-IgG complexes. In a recent pituitary imaging study of patients with macroprolactinemia, abnormal pituitary imaging was found in $21 \%$ (25). These radiological findings in macroprolactinemic patients should, however, be interpreted with caution because of the high incidence of false positives in the normal population and the persistence of a normal pituitary function in many of these patients
(26). On the other hand, we found circulating PRL-IgG complexes in two patients with a stalk-compressing meningioma and in one patient with a histologically proven residual macroprolactinoma. High molecular mass PRL forms which resisted repeated thawing and freezing have been demonstrated previously in the extracts of PRL-secreting pituitary tumors (27). Our finding of an association of macroprolactin with stalk-compressing cerebral tumors and cerebral sarcoidosis warrants a cautious work-up of patients with macroprolactinemia, including an MRI of the pituitary. Taken together, however, our clinical data suggest that macroprolactinemia is usually associated with benign conditions and that the lack of clinical symptoms and endocrinological consequences in hyperprolactinemic patients with only minor pituitary abnormalities on MRI or CT scanning must rather favor the diagnosis of macroprolactinemia-associated hyperprolactinemia.

A reduced biological activity of macroprolactin (compared with monomeric PRL) has been suggested as the reason for lack of symptoms in macroprolactinemic patients $(11,21,23)$. On the other hand, some studies, often based on single-case reports, found that serum containing high molecular mass PRL exhibited lower, higher or similar biological activity compared with serum with monomeric PRL $(11,15,27,28)$. These differences may have a technical origin, or alternatively they might further indicate some heterogeneity of these high molecular mass PRL complexes. We found a difference in the bioactivity pattern of sera from our patients with macroprolactinemia, depending on the nature of the complex. In macroprolactinemic sera without measurable circulating PRL-IgG complexes, bioactivity values increased with increasing free PRL concentrations, but not with total immunoassayable PRL concentrations, suggesting no or limited agonistic activity of these non-IgG related complexes on PRL receptors. In accord with two previously performed bioactivity/immunoreactivity correlation studies, which included only four and seven PRLIgG-containing serum samples, we found that in the samples containing PRL-IgG complexes, the bioactivity values correlated well with the measured total PRL immunoactivity of the sera, suggesting that PRL-IgG complexes have an intrinsic bioactivity $(15,16)$.

Although the bioactivity of the total sera, and not of the PEG precipitated PRL, was measured by us, we think, however, that the total PRL immunoreactivity can be used to represent PRL-IgG complexes, since the majority of PRL was found to be IgG-bound. No difference in bioactivity was found in our study between patient groups with a different initial complaint. Bioactivity in the $\mathrm{Nb} 2$ assay was comparable between patients with idiopathic hyperprolactinemia and those with another origin for their hyperprolactinemia. Therefore, the different biological activities of the circulating PRL-IgG complexes cannot explain 
the absence or presence of clinical symptoms in macroprolactinemic samples.

In conclusion, we confirmed that in the majority of cases of macroprolactinemia, detected by PEG precipitation, are related to PRL-IgG complexes, associated with moderately elevated PRL concentrations if measured by the Elecsys automated immunoassay system. Besides a tendency for a higher frequency of galactorrhea and a higher free PRL level in patients without IgG-containing complexes, no other clinical or hormonal findings were found to differ between macroprolactinemic patients with or without circulating PRL-IgG complexes. Furthermore, these PRLIgG-positive macroprolactinemic patients mostly had free PRL levels within normal ranges, and in about two-thirds of them other conditions were present to account for the complaint. While in none of them was an impact on the pituitary and gonadal function documented, microprolactinoma had been diagnosed on a radiological basis in one-fifth of them. On the other hand, the PRL-IgG complexes were shown to possess a PRL-like biological activity in the $\mathrm{Nb} 2$ assay, which was related to the immunoreactivity, but unrelated to the clinical presentation.

\section{Acknowledgements}

This study was supported by a research grant from the Belgian Study Group for Pediatric Endocrinology and from the Flemish Government (GOA 97-02-04).

\section{References}

1 Smith CR \& Norman MR. Prolactin and growth hormone: molecular heterogeneity and measurement in serum. Annals of Clinical Biochemistry 199027 542-550.

2 Andino NA, Bidot C, Valdes M \& Machado AJ. Chromatographic pattern of circulating prolactin in ovulatory hyperprolactinemia. Fertility and Sterility $1985 \mathbf{4 4} 600-605$.

3 Hattori N. Frequency of macroprolactinemia in pregnant women and the heterogeneity of its etiologies. Journal of Clinical Endocrinology and Metabolism 199681 586-590.

4 Bjoro T, Morkrid L, Wergeland R, Turter A, Kvistborg A, Sand T et al. Frequency of hyperprolactinaemia due to large molecular weight prolactin (150-170 kD PRL). Scandinavian Journal of Clinical and Laboratory Investigation 199555 139-147.

5 Olukoga AO \& Kane JW. Macroprolactinaemia: validation and application of the polyethylene glycol precipitation test and clinical characterization of the condition. Clinical Endocrinology 199951 119-126.

6 Sanchez-Eixeres MR, Mauri M, Alfayate R, Graells ML, Miralles C, Lopez A et al. Prevalence of macroprolactin detected by ElecSys 2010. Hormone Research 200156 87-92.

7 Fahie-Wilson MN \& Soule SG. Macroprolactinaemia: contribution to hyperprolactinaemia in a district general hospital and evaluation of a screening test based on precipitation with polyethylene glycol. Annals of Clinical Biochemistry 199734 252-258.

8 Fahie-Wilson M, Brunsden P, Surrey J \& Everitt A. Macroprolactin and the Roche Elecsys prolactin assay: characteristics of the reaction and detection by precipitation with polyethylene glycol. Clinical Chemistry 200046 1993-1995.
9 Leslie H, Courtney CH, Bell PM, Hadden DR, McCance DR, Ellis PK et al. Laboratory and clinical experience in 55 patients with macroprolactinemia identified by a simple polyethylene glycol precipitation method. Journal of Clinical Endocrinology and Metabolism $2001862743-2746$.

10 Schiettecatte J, De Schepper J, Velkeniers B, Smitz J \& Van Steirteghem A. Rapid detection of macroprolactin in the form of prolactin-immunoglobulin $\mathrm{G}$ complexes by immunoprecipitation with anti-human IgG-agarose. Clinical Chemistry and Laboratory Medicine 200139 1244-1248.

11 Jackson RD, Wortsman J \& Malarkey WB. Characterization of a large molecular weight prolactin in women with idiopathic hyperprolactinemia and normal menses. Journal of Clinical Endocrinology and Metabolism $198561258-264$.

12 Carlson HE, Markoff E \& Lee DW. On the nature of serum prolactin in two patients with macroprolactinemia. Fertility and Sterility $1992 \mathbf{5 8} 78-87$.

13 Hattori N, Ikekubo K, Ishihara T, Moridera K, Hino M \& Kurahachi H. A normal ovulatory woman with hyperprolactinemia: presence of anti-prolactin autoantibody and the regulation of prolactin secretion. Acta Endocrinologica 1992 126 497-500.

14 Bonhoff A, Vuille JC, Gomez F \& Gellersen B. Identification of macroprolactin in a patient with asymptomatic hyperprolactinemia as a stable PRL-IgG complex. Experimental and Clinical Endocrinology and Diabetes 1995103 252-255.

15 Leite V, Cosby H, Sobrinho LG, Fresnoza MA, Santos MA \& Friesen HG. Characterization of big, big prolactin in patients with hyperprolactinaemia. Clinical Endocrinology $1992 \quad 37$ 365-372.

16 Hattori N, Ikekubo K, Ishihara T, Moridera K, Hino M \& Kurahachi H. Correlation of the antibody titers with serum prolactin levels and their clinical course in patients with anti-prolactin autoantibody. European Journal of Endocrinology $1994130438-445$.

17 Guay AT, Sabharwal P, Varma S \& Malarkey WB. Delayed diagnosis of psychological erectile dysfunction because of the presence of macroprolactinemia. Journal of Clinical Endocrinology and Metabolism 199681 2512-2514.

18 Rowe RC, Cowden EA, Faiman C \& Friesen HG. Correlation of Nb2 bioassay and radioimmunoassay values for human serum prolactin. Journal of Clinical Endocrinology and Metabolism 1983 $57942-946$.

19 Vieira JG, Tachibana TT, Obara LH \& Maciel RM. Extensive experience and validation of polyethylene glycol precipitation as a screening method for macroprolactinemia. Clinical Chemistry $1998441758-1759$.

20 Vallette-Kasic S, Morange-Ramos I, Selim A, Gunz G, Morange S, Enjalbert A et al. Macroprolactinemia revisited: a study on 106 patients. Journal of Clinical Endocrinology and Metabolism 2002 $87581-588$.

21 Fraser IS, Lun ZG, Zhou JP, Herington AC, McCarron G, Caterson I et al. Detailed assessment of big big prolactin in women with hyperprolactinemia and normal ovarian function. Journal of Clinical Endocrinology and Metabolism 198969 585-592.

22 Cavaco B, Leite V, Santos MA, Arranhado E \& Sobrinho LG. Some forms of big big prolactin behave as a complex of monomeric prolactin with an immunoglobulin $\mathrm{G}$ in patients with macroprolactinemia or prolactinoma. Journal of Clinical Endocrinology and Metabolism $1995802342-2346$.

23 Pereira MC, Sobrinho LG, Afonso AM, Ferreira JM, Santos MA \& Sousa MF. Is idiopathic hyperprolactinemia a transitional stage toward prolactinoma? Obstetrics and Gynecology $1987 \mathbf{7 0}$ 305-308

24 Sluijmer AV \& Lappohn RE. Clinical history and outcome of 59 patients with idiopathic hyperprolactinemia. Fertility and Sterility $19925872-77$.

25 Hauache OM, Rochat AJ, Maia ACM, Maciel RMB \& Vieira JG. Screening for macroprolactinemia and pituitary imaging studies. Clinical Endocrinology 200257 327-331. 
26 Hall WA, Luciano MG, Doppman JL, Patronas NJ \& Oldfield EH. Pituitary magnetic resonance imaging in normal human volunteers: occult adenomas in the general population. Annals of Internal Medicine 1994120 817-820.

27 Ohnami S, Eto S, Ohnami S, Soejima T \& Nakata H. Characterization of 'big big prolactin' in serum and tumor extract in patients with PRL-secreting tumor. Endocrinology 198734 $325-334$.
28 Hattori N \& Inagaki C. Anti-prolactin (PRL) autoantibodies cause asymptomatic hyperprolactinemia: bioassay and clearance studies of PRL-immunoglobulin G complex. Journal of Clinical Endocrinology and Metabolism 199782 3107-3110.

Received 11 May 2003

Accepted 1 July 2003 